\title{
ETHOS: Miniature Orientation Sensor for Wearable Human Motion Analysis
}

\author{
Holger Harms *, Oliver Amft*, $\dagger$, Rene Winkler, Johannes Schumm*, Martin Kusserow*, and Gerhard Troester* \\ *Wearable Computing Lab., ETH Zurich, Switzerland \\ $\dagger$ ACTLab, Signal Processing Systems, TU Eindhoven, The Netherlands \\ Email: \{harms, amft, schumm, kusserow, troester\}@ife.ee.ethz.ch
}

\begin{abstract}
Inertial and magnetic sensors offers a sourceless and mobile option to obtain body posture and motion for personal sports or healthcare assistants, if sensors could be unobtrusively integrated in casual garments and accessories. We present in this paper design, implementation, and evaluation results for a novel miniature attitude and heading reference system (AHRS) named ETHOS using current off-the-shelf technologies.

ETHOS has a unit size of $2.5 \mathrm{~cm}^{3}$, which is substantially below most currently marketed attitude heading reference systems, while the unit contains processing resources to estimate its orientation online. Results on power consumption in relation to sampling frequency and sensor use are presented. Moreover two sensor fusion algorithms to estimate orientation: a quaternionbased Kalman-, and a complementary filter. Evaluations of orientation estimation accuracy in static and dynamic conditions revealed that complementary filtering reached sufficient accuracy while consuming $46 \%$ of a Kalman's power. The system runtime of ETHOS was found to be $\mathbf{1 0}$ hours at a complementary filter update rate of $128 \mathrm{~Hz}$. Furthermore, we found that a ETHOS prototype functioned with a sufficient accuracy in estimating human movement in real-life conditions using an arm rehabilitation robot.
\end{abstract}

Keywords-ETH orientation sensor, inertial measurement unit

\section{INTRODUCTION}

Body posture and motion is a vital source of information for various applications in sports, physical rehabilitation and biomechnanical function assessment, as well as in ergonomics, gaming, and movie production. Human posture and motion information is used in these fields to augment the user's perception, e.g. to train skills while cuing motivation [1]. Alternatively, information is embedded into virtual or cross reality representations, e.g. for gaming entertainment purposes [2]. Acquiring posture and motion information is a key challenge in many of the aforementioned fields, which can substantially limit applications. For example, in many functional motion assessments monitoring should be continuously performed, body motion should not be restricted, and recording equipment must be mobile in order to be used outside of laboratory environments.

Inertial and magnetic sensing concepts that can be attached to the body fulfill mobility requirements since they operate sourceless, hence do not require stationary installations to function. Thus, monitoring of postures and motion in natural real-life settings could become feasible. Inertial sensors, as accelerometers and gyroscopes, can be constructed using the widely adopted MEMS technology and therefore small device outlines and low weight systems could be achieved. To this end, uni-modal inertial sensors have been unobtrusively integrated into textiles and commonly used clothing, e.g. in [3]. However, accurate orientation estimation and capturing dynamic body motions in three dimensions requires a combination of sensors. Typically three accelerometers measuring linear accelerations, three rate gyroscopes sensing angular velocity, and three magnetometers that detect the earth magnetic field are used. In such attitude and heading reference systems (AHRS), every sensor is placed orthogonally to others of the same modality to provide measurements along each axis [4]-[7].

AHRS allow to compute their orientation and thus orientation of body movement when attached to the body. Multiple units can be used form a system measuring joint body segment orientations. However, size, outline, weight, and computational requirements are critical and yet unresolved challenges for continuous mobile and wearable use of AHRS.

In this work we address these challenges by designing and evaluating a novel miniature AHRS unit, named ETH Orientation Sensor (ETHOS). ETHOS was specifically designed for wearable use. It uses current off-the-shelf sensor components were integrated into a system offering local processing resources, e.g. to compute orientation online. The system offers both, local data storage and ultra-low power wireless transmission options, and scalable processing capacity which can be adapted to the application's demands, e.g. regarding orientation streaming bandwidth.

This paper provides the following contributions:

1) We present the ETHOS system architecture based on current off-the-shelf sensor component and integration technologies, with which a PCB form factor of $14 \times 45 \times 4 \mathrm{~mm}^{3}$ and weight (without battery) of $4.2 \mathrm{~g}$.

2) ETHOS was evaluated regarding its continuous runtime and energy consumptions with respect to sensor sampling frequency and orientation estimation performance. For this purpose, orientation estimation using Kalman and linear filters are compared.

3) We studied orientation estimation accuracy in a reallife application using an arm rehabilitation robot. In this analysis, orientation of the upper body was estimated and compared to the commercial Xsens system [7]. 


\section{SYSTEM ARCHITECTURE}

ETHOS comprises five principle design blocks, (1) the main processor, (2) sensors, (3) power conversion and battery charger, (4) communication interfaces, and (5) system memory. The front and backside of the system are depicted in Figure 1.
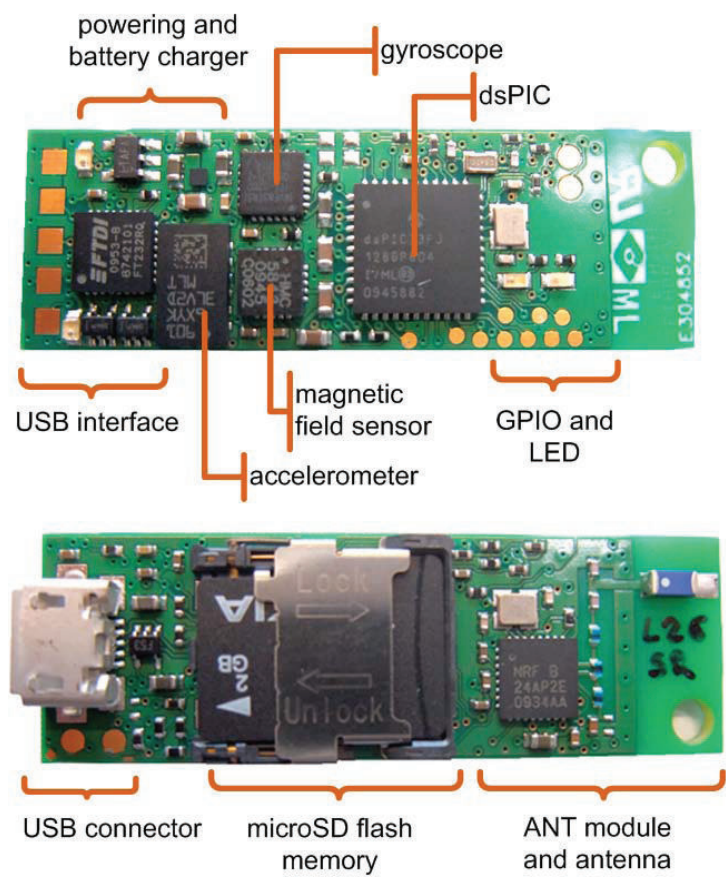

Fig. 1. Front and backside of ETHOS units and installed components.

The final ETHOS unit resulted in WxLxH dimensions of $14 \times 45 \times 4 \mathrm{~mm}^{3}$. We implemented the unit in an rectangular shape to support attachment along body limbs. The system width was imitated by the width of the microSD socket, the height is given by heights of ICs and PCB thickness.

Figure 2(a) depicts a housing implementation with $\mathrm{WxLxH}$ dimensions of $24 \times 46 \times 14 \mathrm{~mm}^{3}$, where the ETHOS PCB and battery are stacked. In a bracelet-like design with a side-byside alignment of the PCB and battery, a maximum height of $<10 \mathrm{~mm}$ was achieved (see Fig. 2(b)).

\section{A. Main processor}

The central processing unit of ETHOS is a 16-bit dsPIC (MICROCHIP, dsPIC33FJ128). The dsPIC allows computation of maximum 40 MIPS at low power consumption, and provides sufficient on-board RAM $(16 \mathrm{kB})$ for operation of filter algorithms. Moreover, it features an integrated real time clock / calendar, which is crucial for tagging of data and synchronization of multiple ETHOS units.

\section{B. Onboard sensors}

Orientation information can be inferred by fusion of inertial and magnetic field sensor data. The system gathers the individual information by three MEMS devices: an accelerometer,

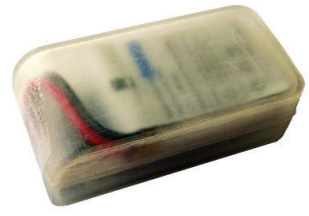

(a) Stacked system case

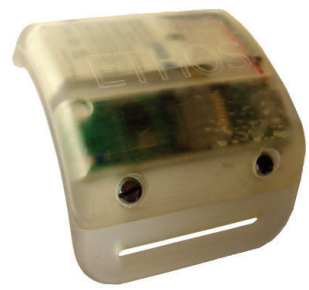

(b) Bracelet system case
Fig. 2. Two system cases containing each one ETHOS PCB and a battery. The left picture shows a stacked system housing which minimizes the volume. The right picture depicts a bracelet-like housing, which is optimized to be unobtrusively worn for long periods of time.

a gyroscope and a magnetic field sensor. Moreover a temperature sensor and system power monitor are implemented for automatic self-calibration. All sensors were selected to have all three axes available and a digital interface to avoid analog line filter circuitry.

We defined standard sampling frequencies of sensors to 1 , 16, 32, 64 and $128 \mathrm{~Hz}$

Accelerometer: A three-axis accelerometer (Linear Technology, LIS3LV02DL) can be configured to resolve $\pm 2 / 6 \mathrm{~g}$ with a 16-bit resolution. For inference of orientation a maximum resolution of $1 \mathrm{~g}$ is sufficient. Hence, at a configuration of $2 \mathrm{~g}$, a theoretical resolution of $0.06 \mathrm{mg} / \mathrm{LSB}$ is achieved.

Magnetic field sensor: Earth magnetic is sensed by an integrated digital compass IC (Honeywell, HMC5843) in all three axes. The devices has a maximum measurement range of $\pm 4 \mathrm{Ga}$ and a resolution of 12-bit. We configured the device to operate at a maximum measurement range of $1 \mathrm{Ga}$, as the earth magnetic field ranges from approximately $0.31-0.58 \mathrm{Ga}$.

Gyroscope and temperature sensor: A three-axes gyroscope (Invensense, ITG-3200) allows sensing of the rate of change with a maximum measurement range of $2000^{\circ} / \mathrm{s}$ at resolution of 16-bit. In a worst case scenario, the zerorate (bias) could deviate about $40 \%$ s over the specified temperature range of the device. Hence, an integrated temperature sensor is installed for automatic calibration of the zero-rate.

\section{Communication interfaces}

ANT module: Each board comprises an 8-channel ultra low power ANT module for wireless communication. ANT allows interconnection of multiple ETHOS units for creation of wireless body area networks (BAN). This network could be extended by other ANT or ANT+ enabled devices, e.g. a heart rate belt, step-counter, or GPS module. Moreover, it allows to interface the unit with mobile computers. While the ANT standard is impractical for streaming of raw data, the bandwidth would allow a transmission of gathered orientation data at a maximum frequency of up to $200 \mathrm{~Hz}$. The basic design of the ANT circuitry is based on the BodyANT modules [8].

USB interface: An USB interface allows bi-directional transmission of data to external hardware. In a live-mode, ETHOS allows transmission of gathered data to an external 
TABLE I

STATIC RMS NOISE OF INDIVIDUAL SENSOR AXES AT A SAMPLING FREQUENCY OF $128 \mathrm{~Hz}$.

\begin{tabular}{|c||c|c|c|}
\hline \multicolumn{1}{|c||}{} & \multicolumn{3}{c|}{ Axis } \\
Sensor & $\mathrm{X}$ & $\mathrm{Y}$ & $\mathrm{Z}$ \\
\hline \hline Acceleration $[\mathrm{mg}]$ & 0.3 & 0.16 & 3.2 \\
\hline Magnetic field $[\mathrm{mGa}]$ & 2 & 1.7 & 2 \\
\hline Gyroscope $[\% \mathrm{~s}]$ & 1 & 1.1 & 1 \\
\hline \hline
\end{tabular}

PC for live- display or further analysis. In a configuration mode, previously gathered data can be read out from onboard memory. The maximum transmission rate is $366 \mathrm{kB} / \mathrm{s}$.

\section{System power}

System power is provided by an external lithium-polymer battery with a nominal voltage of $3.7 \mathrm{~V}$. A miniature onboard LDO provides a constant system voltage of $3.3 \mathrm{~V}$ and a maximum current $150 \mathrm{~mA}$. If the system is connected to an USB port the system battery is loaded by an integrated LiIon battery charger. The system supervises system power and indicates low power by an LED. In our standard configuration we use a miniature $300 \mathrm{mAh}$ battery with $\mathrm{WxLxH}$ dimensions of $20 \times 30 \times 3 \mathrm{~mm}^{3}$.

\section{E. System memory}

A microSD card slot was installed to store data, timestamps and system configuration on non-volatile flash memory. microSD flash memory is of small dimensions, available with high capacities and can be easily replaced in case of low memory. A sample-and-store mode results in a maximum data volume of $10 \mathrm{MB} / \mathrm{h}$. Hence, every installed GB of flash memory covers approximately 4 days of continuously sampled data, time and calendar information.

\section{DEVICE CHARACTERIZATION}

\section{A. Sensing accuracy}

The noise of individual sensors determines the system accuracy, if no additional filter is applied. Table I summarizes the RMS of each individual sensor at a maximum sampling frequency of $128 \mathrm{~Hz}$. The measurements indicate stable sensor operation and low sensor noise in static conditions.

\section{B. Power consumption and system runtime}

The overall system runtime is defined by (1) the battery, and individual consumption of the sensors, processor, and periphery. In idle mode, where the processor operates at $8 \mathrm{MHz}$ and sensors are in sleep mode, we measured a minimum power consumption of $17 \mathrm{~mA}$. Further measurements showed that every additional MIPS consumes $1 \mathrm{~mA}$. After enabling of sensors, the system can enter a sample-and-store $(\mathrm{SaS})$ mode. In $\mathrm{SaS}$ mode, sampled from individual sensors and stored to the local memory. The SaS mode can be run at a minimum processor clock frequency of $8 \mathrm{MHz}$ for the maximum sampling frequencies up to $128 \mathrm{~Hz}$. Hence the required
TABLE II

POWER CONSUMPTION OF INDIVIDUAL SENSORS AT DEFINED SAMPLING FREQUENCIES. SENSORS ARE OPERATED AT A VOLTAGE OF $3.3 \mathrm{~V}$.

\begin{tabular}{|c||c|c|c|c|c|}
\hline \multicolumn{1}{|c||}{} & \multicolumn{5}{c|}{ Sampling frequency [Hz] } \\
Sensor & 1 & 16 & 32 & 64 & 128 \\
\hline \hline Acceleration [mA] & 0.7 & 0.9 & 1.2 & 1.7 & 2.6 \\
\hline Magnetic field [mA] & 1.1 & 1.1 & 1.2 & 1.4 & 1.8 \\
\hline Gyroscope [mA] & 6.4 & 6.5 & 6.6 & 6.8 & 7.3 \\
\hline \hline
\end{tabular}

power depends on the consumption of sensors at specific sampling frequencies. Table II summarizes the consumption of sensors at defined sampling frequencies and constant system voltage of $3.3 \mathrm{~V}$. The measurements indicate a specifically low consumption of the acceleration sensor (max. $2.6 \mathrm{~mA}$ ) and magnetic field sensor (max. $1.8 \mathrm{~mA}$ ), while the gyroscope ends up in a relatively higher consumption (max. $7.3 \mathrm{~mA}$ ).

Figure 3 summarizes power consumption and corresponding runtime of ETHOS at defined sampling frequencies. The results indicate a sufficient operation time to enable long-term recordings of $>10$ hours. If longer run-times are required, the current miniature battery could be replaced by an alternative with larger capacity.

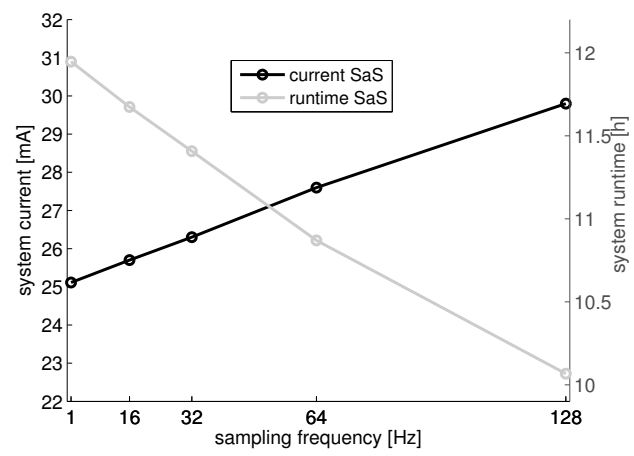

Fig. 3. Power consumption (black) and system runtime (gray) for a 300mAh battery at different sampling frequencies.

\section{ALGORITHMS FOR COMPUTATION OF ORIENTATION}

Orientation of a rigid body in a 3-dimensional Euclidean space can be described by a set of three Euler angles, denoted by $\Phi$ (roll angle), $\Theta$ (pitch angle), and $\Psi$ (yaw angle). Computation of orientation means that a sensor calculates three angles around the axes of it's local frame must be rotated to be aligned with a global reference frame (typically the earth). Figure 4(a) illustrates the reference frame as it was defined on ETHOS.

\section{A. Algorithms for computation of orientation}

Orientation from raw sensor data: Plain computation of Euler angles from raw sensor data can be performed without application of additional filter [9]. In this approach, acceleration data is used to infer the roll angle $\Phi$ and pitch 
angle $\Theta$, while the yaw angle $\Psi$ requires further information of the magnetic field sensor. While this approach is feasible for static postures, it is impractical in cases of dynamics. Low computational requirements allow operation of the dsPIC at the minimum mode of $8 \mathrm{MHz}$. Hence, for this basic mode the power consumption and system runtime are equivalent as for the sample-and-store mode (compare Fig. 3. However, if dynamic movements are tracked additional filter are required for reliable inference of orientation.

Complementary filter design: Complementary filter exploit the complementary spectral characteristics of noisy signals to compute orientation [10]. While complementary filter can be operated at low computational costs, it has been shown that they are capable to track orientation of human body limbs with a sufficient accuracy [4]. We implemented each one Euler based complementary filter framework for online computation of orientation on an ETHOS prototype platform, and offline analysis of data in MATLAB.

Kalman filter design: A Kalman filter is a set of mathematical equations that estimates the state of a process in an optimal least-squares sense. As the filter is operated recursively, it becomes suitable for many real-time applications, like motion tracking. Here, the state is e.g. the estimated orientation, and the process is the human motion. While Euler-based Kalman tracking filter require $n=6$ states, they suffer from singularity problems. However, we implemented a quaternionbased $n=7$ state extended Kalman filter as proposed in literature [11], [12] in MATLAB. While the computational complexity of Kalman filter increases with the number of states in an order of $O\left(n^{3}\right)$, a quaternion operation omits trigonometric functions as they are required in Euler-based approaches.

\section{B. Orientation accuracy}

To determine orientation errors, we used an early prototype of ETHOS that comprises the same sensing and processing architecture, see Figure 4(b).

Static orientation error: The static orientation error of our online complementary filter implementation was evaluated against an in-house fabricated absolute goniometer reference device. We mounted the sensor inside the reference device and manipulated the roll, pitch, and yaw angles independently in steps of $5^{\circ}$ in the full range of $360^{\circ}$. The average RMS error of the complementary filter was $0.5^{\circ}$ for the roll angle $\Phi, 1.3^{\circ}$ for the Pitch angle $\Theta$, and $1.8^{\circ}$ for the Yaw angle $\Psi$.

Orientation error in case of dynamics: We mounted an ETHOS prototype platform on a standard Xsens MT9 sensor [7] for evaluation of individual accuracy of both filter approaches. The setup is depicted in Figure 4(b). While specific dynamics motions were emulated, simultaneously recorded data of both systems was transfered to a PC and stored for subsequent offline analysis. After application of the complementary and Kalman filter to the sensor data, the resulting orientations were compared to the reference data in order to compute the individual RMS error.

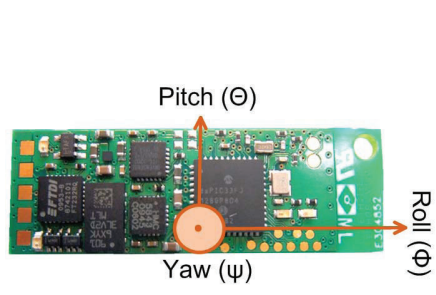

(a) Defined Euler angles on ETHOS

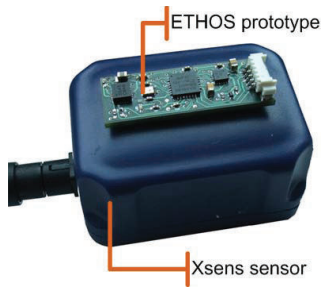

(b) ETHOS prototype on Xsens reference sensor
Fig. 4. Right: ETHOS prototype unit mounted on a Xsens reference sensor for evaluation of measurement accuracy.

TABLE III

RMS ERROR OF COMPLEMENTARY AND KALMAN FILTER IMPLEMENTATIONS DURING INDUCTION OF UNI-AXIAL ACCELERATION AND ROTATION.

\begin{tabular}{|c|c||c|c|c|}
\hline & \multicolumn{1}{|c||}{} & \multicolumn{3}{c|}{ RMS error } \\
& Filter & $\Phi$ & $\Theta$ & $\Psi$ \\
\hline \hline Accel. & Compl. & $0.9^{\circ}$ & $1.9^{\circ}$ & $3.5^{\circ}$ \\
\hline & Kalman & $0.3^{\circ}$ & $1.3^{\circ}$ & $2^{\circ}$ \\
\hline \hline Rot. & Compl. & $1.4^{\circ}$ & $0.9^{\circ}$ & $1.3^{\circ}$ \\
\hline & Kalman & $2.1^{\circ}$ & $0.9^{\circ}$ & $1.4^{\circ}$ \\
\hline \hline
\end{tabular}

We evaluated the filter performance in two possible cases of random dynamics that could occur and impair accuracy: (1) random uni-axial acceleration with a maximum amplitude of $20 \mathrm{~m} / \mathrm{s}^{2}$, and (2) random uni-axial rotations in a goniometer with a maximum rate of $150 \%$ s. All tests were performed axiswise for $4-5 \mathrm{~s}$ and repeated for three times. Table III summarizes the RMS error of orientation.

The results show a lower RMS errors for the Kalman filter estimations than for the complementary filter. However, we conclude that the performance of the complementary filter is still sufficient for tracking of human motions. Hence, it's online implementation on ETHOS should work reliably for dynamics as they are expected in human movements.

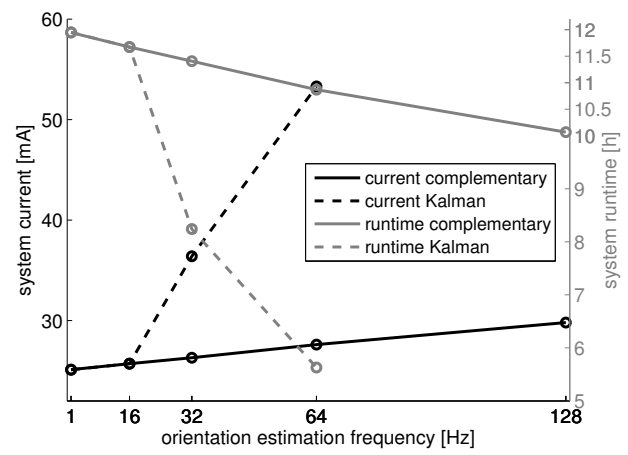

Fig. 5. Power consumption (black) and system runtime (gray) of the complementary filter (straight line) and Kalman filter (dashed line) at different update rates. 

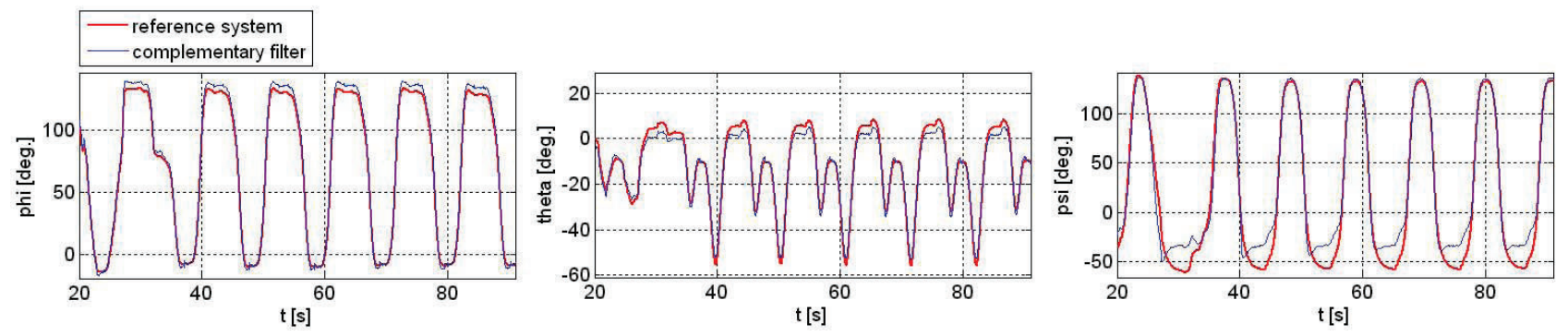

Fig. 6. Comparison of complementary filter and reference system outputs during execution of arm motions.

\section{Power consumption and system runtime}

In our MATLAB implementation of the Kalman filter, we counted in total 3357 floating point operations per orientation estimation. As the dsPIC on ETHOS is not equipped with a floating point unit (FPU), the operations are translated by a mathematical library to multiple fixed point operations. Based on the library documentation and related work [13] we estimated an average of 145 instruction cycles to execute one FLOP. Hence, the dsPIC needs to perform about $486 \mathrm{k}$ cycles for every estimation of orientation. The available computational power of 40MIPS on ETHOS would be sufficient for 80 estimations per second. In practice, the estimations would be restricted to $64 \mathrm{~Hz}$, as additional processing power is required for $\mathrm{I} / \mathrm{O}$ operations. However, computational optimization could reduce the required FLOPS per computed orientation significantly [14]. Figure 5 depicts the estimated power consumption of an online Kalman filter implementation and according runtime utilizing a battery of $300 \mathrm{mAh}$. The plot considers estimations of up to $64 \mathrm{~Hz}$, where a system runtime of 5.5 hours is predicted.

However, our online complementary filter implementation is capable to compute Euler-angle orientation for all defined update rates of up to $128 \mathrm{~Hz}$ at a minimum processor clock frequency of $8 \mathrm{MHz}$. As indicated in Figure 5, a system runtime of up to 12 hours is achieved.

The results indicate, that a complementary filter implementation requires $46 \%$ of the computational power at an update rate of $64 \mathrm{~Hz}$.

\section{VALIDATION OF THE SYSTEM}

We evaluated the effectiveness of our online filter implementation for realistic human motions in a case study. For this purpose, we monitored arm motions of a subject performing exercises with an ARMin arm rehabilitation robot [15]. To quantify accuracy, we again mounted an ETHOS prototype to a Xsens reference (compare Fig. 4(b)). Both units were attached to the arm of a healthy subject, as shown in Figure 7. In total, six rehabilitation exercises were performed by the ARMin robot. ARMin was operated in the teach-and-repeat mode. In a preparing teach step, the therapist performed each of the six rehabilitation exercises by guiding the patient's arm, while the robot recorded the motion path. Subsequently, the subject repeated the motion five times, while the robot supervised and supported the execution.

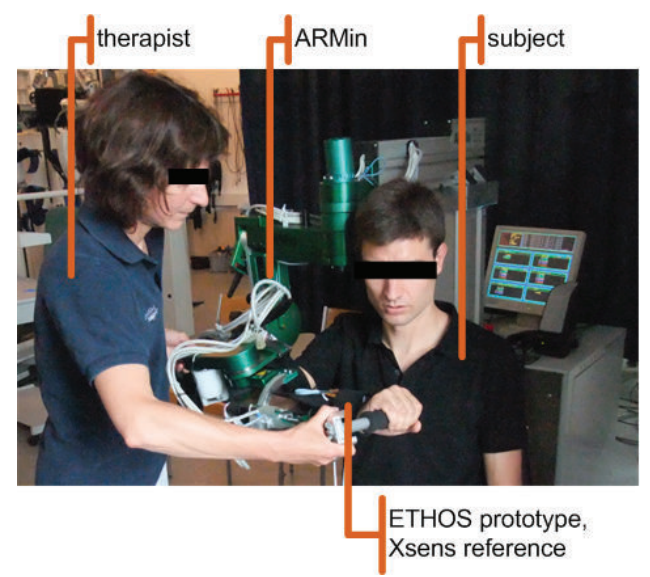

Fig. 7. Experiment setup for evaluating the complementary filter implementation. Actual arm rehabilitation exercises were executed by a subject, supervised by the ARMin rehabilitation robot.

Figure 6 shows the computed orientation using our online complementary filter and the reference Xsens output for an exemplary motion. The graph indicates that exercises were repeated in a similar way, while the residuum between both orientations was small. The overall RMS error of the complementary filter for all six exercises and each of the five repetitions was $6.1^{\circ}$ for the roll angle $\Phi, 2.6^{\circ}$ for the pitch angle $\Theta$, and $18.1^{\circ}$ for the Yaw angle $\Psi$. We observed relatively large deviations for the Yaw angle $\Psi$, especially at negative angles. At those positions, the arm was moved close to the ARMin robot, where metal and alternating currents created magnetic field disturbances. The Yaw angle was specifically affected by this fields, as it is the only Euler angle that is derived with the help of magnetic field sensor data. While it remains unclear to which extend the reference Xsens sensor was affected, a precedent system calibration of the sensor unit could improve accuracy.

\section{CONCLUSION}

We introduced in this work ETHOS, a miniature attitude and heading reference system (AHRS) that can be unobtrusively integrated into causal garments or accessories. ETHOS 
was implemented in an elongated shape with final $\mathrm{WxLxH}$ dimensions of $14 \times 45 \times 4 \mathrm{~mm}^{3}$.

An evaluation of the system noise at selected sampling frequency of up to $128 \mathrm{~Hz}$ showed a reliable function of the system. In sample-and-store mode, the system runtime was found to be about 10 hours at $128 \mathrm{~Hz}$ sampling frequency. Moreover, we evaluated two sensor fusion algorithms to estimate orientation: a quaternion-based Kalman filter, and a complementary filter. Evaluations of the orientation estimation in static and dynamic conditions revealed that a complementary filter operates at sufficient accuracy for the dynamics expected in human movements. ETHOS was shown to be applicable for tracking human movements under real-life conditions in a study with an arm rehabilitation robot. The power consumption of the complementary filter was determined to be $46 \%$ lower than that of a Kalman filter. The system runtime of ETHOS was found to be 10 hours at a complementary filter update rate of $128 \mathrm{~Hz}$, and 5.5 hours for a Kalman filter with an update rate of $64 \mathrm{~Hz}$.

We expect that miniature design and power-efficient operation of ETHOS will permit its application in further studies.

\section{REFERENCES}

[1] E. Vergaro, M. Casadio, V. Squeri, P. Giannono, P. Morasso, and V. Sanguineti, "Self-adaptive robot training of stroke survivors for continuous tracking movements," Journal of NeuroEngineering and Rehabilitation, vol. 7.

[2] D. Bannach, O. Amft, K. Kunze, E. Heinz, G. Troster, and P. Lukowicz, "Waving real hand gestures recorded by wearable motion sensors to a virtual car and driver in a mixed-reality parking game," in IEEE Symposium on Computational Intelligence and Games, 2007. CIG 2007, 2007, pp. 32-39.

[3] H. Harms, O. Amft, D. Roggen, and G. Tröster, "Rapid prototyping of smart garments for activity-aware applications," Journal of Ambient Intelligence and Smart Environments (JAISE), 2009.
[4] A. Young, M. Ling, and D. Arvind, "Orient-2: A realtime wireless posture tracking system using local orientation estimation," in Proceedings of the 4th workshop on Embedded networked sensors. ACM, 2007, p. 57.

[5] E. Bachmann, X. Yun, D. McKinney, R. McGhee, and M. Zyda, "Design and Implementation of MARG Sensor for 3-DOF Orientation Measurement of Rigid Bodies," in IEEE International Conference on Robotics and Automation, vol. 1, 2003, pp. 1171-1178.

[6] A. Lynch, B. Majeed, B. O’Flynn, J. Barton, F. Murphy, K. Delaney, and S. O'Mathuna, "A wireless inertial measurement system (WIMS) for an interactive dance environment," in Journal of Physics: Conference Series, vol. 15. IOP Publishing, 2005, p. 95.

[7] Xsens, "3DOF Orientation Tracker MTx - Xsens," http://www.xsens.com/en/general/mtx, 2010, [Online; last accessed 28-July-2010].

[8] M. Kusserow, O. Amft, and G. Tröster, "Bodyant: Miniature wireless sensors for naturalistic monitoring of daily activity," in Proceedings of the Fourth International Conference on Body Area Networks, 2009.

[9] C. Liu, Z. Zhou, and X. Fu, "Attitude Determination for MAVs Using a Kalman Filter," Tsinghua Science \& Technology, vol. 13, no. 5, pp. 593-597, 2008.

[10] E. Bachmann, "Inertial And Magnetic Tracking Of Limb Segment Orientation For Inserting Humans Into Synthetic Environments," Ph.D. Dissertation, Naval Postgraduate School, 2000.

[11] X. Yun and E. Bachmann, "Design, implementation, and experimental results of a quaternion-based Kalman filter for human body motion tracking," IEEE Transactions on Robotics, vol. 22, no. 6, pp. 12161227, 2006.

[12] J. Marins, X. Yun, E. Bachmann, R. McGhee, and M. Zyda, "An extended Kalman filter for quaternion-based orientation estimation using MARG sensors," in Proceedings of the 2001 IEEE/RSJ, International Conference on Intelligent Robots and Systems, Maui, Hawaii, 2001.

[13] H. Luinge, "Inertial sensing of human movement," 2002.

[14] M. Grewal and A. Andrews, Kalman filtering: theory and practice using MATLAB. Wiley-IEEE Press, 2008.

[15] ETHZ, "ETH - Sensory-Motor Systems Lab - Arm Rehabilitation," http://www.sms.mavt.ethz.ch/research/projects/armin/therapy, $\quad 2010$, [Online; last accessed 28-July-2010]. 